\title{
Stability of Runge-Kutta-Pouzet methods for Volterra integro-differential equations with delays*
}

\author{
Chengming HUANG ${ }^{1}$, Stefan VANDEWALLE ${ }^{2}$ \\ 1 School of Mathematics and Statistics, Huazhong University of Science and Technology, \\ Wuhan 430074, China \\ 2 Department of Computerscience, Katholieke Universiteit Leuven, Celestijnenlaan 200A, \\ B3001 Leuven, Belgium
}

(C) Higher Education Press and Springer-Verlag 2009

\begin{abstract}
This paper is concerned with the study of the stability of RungeKutta-Pouzet methods for Volterra integro-differential equations with delays. We are interested in the comparison between the analytical and numerical stability regions. First, we focus on scalar equations with real coefficients. It is proved that all Gauss-Pouzet methods can retain the asymptotic stability of the analytical solution. Then, we consider the multidimensional case. A new stability condition for the stability of the analytical solution is given. Under this condition, the asymptotic stability of Gauss-Pouzet methods is investigated.
\end{abstract}

Keywords Volterra delay integro-differential equation, asymptotic stability, Runge-Kutta-Pouzet method

MSC 65L20, 65R99

\section{Introduction}

Many phenomena in science and engineering can be modelled by initial value problems for functional differential equations. When solving such equations numerically, the question arises as to whether the stability properties of the continuous problem are retained by the discretization. A typical procedure is then to select a representative class of model problems, and to study the stability properties of the continuous solution and of the numerical solution as a function of the model problem parameters. A basic model problem in

* Received March 30, 2008; accepted November 15, 2008

Corresponding author: Chengming HUANG, E-mail: chengming_huang@hotmail.com 
this field is the discrete delay equation

$$
y^{\prime}(t)=\alpha y(t)+\beta y(t-\tau),
$$

where $\alpha, \beta \in \mathbb{R}$ and $\tau$ is a fixed positive number. A very large body of results have been obtained for this equation, see, e.g., Refs. [3,4,7,8,10-14,26,27,29]. Relevant to the case of the distributed delay equation

$$
y^{\prime}(t)=\alpha y(t)+\gamma \int_{t-\tau}^{t} y(\nu) \mathrm{d} \nu,
$$

however, only few results on numerical stability have been published. Baker and Ford [2] investigated the analytical stability region of (1.2) and numerically showed that some linear multistep methods combined with an appropriate quadrature rule are highly stable, with a stability region that approximates the analytical stability region. Luzyanina, Engelborghs and Roose [25] recently gave some stability results of multistep methods under a sufficient condition for the stability of (1.2). Huang and Vandewalle [19] considered a more general equation than (1.2), i.e.,

$$
y^{\prime}(t)=\alpha y(t)+\beta y(t-\tau)+\gamma \int_{t-\tau}^{t} y(\nu) \mathrm{d} \nu,
$$

and proved that the repeated trapezium rule retains the asymptotic stability of (1.3). Wu and Gan [30] further extended the above study to the case of neutral equations.

A natural question that follows, and which will be studied in the present paper, is the stability of high order Runge-Kutta methods, especially collocation methods, for scalar distributed delay equations of type (1.3). Another important problem is the investigation of the stability of multidimensional systems. Unfortunately, at present, there are no explicit conditions known which are suitable to describe the complete, i.e., delaydependent stability region of the latter. One normally resorts to the use of some sufficient conditions for stability of the differential equation system to analyze the stability of numerical methods. For example, in't Hout [20], Hu and Mitsui [17], and Koto [21] studied the stability of Runge-Kutta methods for systems of discrete delay differential equations of type (1.1). In the case of distributed delay equations, Koto [22] recently proved that every $A$-stable natural Runge-Kutta method of Pouzet type can preserve the delay-independent stability of the underlying system. Huang [18] obtained a similar result for linear multistep methods of Pouzet type. Zhao et al. [32], and Zhang and Vandewalle [31] gave some stability conditions for neutral delay-integro-differential equations and investigated the stability of several classes of numerical methods under those conditions. For stability analysis of general Volterra functional differential equations, we refer the reader to Ref. [23].

This paper is organized as follows. In Section 2, we first consider the delay-dependent stability of Runge-Kutta-Pouzet methods of high order for 
distributed delay differential equations. We will prove that all Gauss-Pouzet methods retain the asymptotic stability of (1.3). We also show that the implicit Euler-Pouzet rule violates this property. The latter is different from the case of discrete delay equations. Next, in Section 3, we turn our attention to the case of higher dimensions. A new stability condition for the analytical solution is derived. Under this condition, the asymptotic stability of RungeKutta-Pouzet methods is studied. In Section 4, some numerical examples are given. We end in Section 5 with some concluding remarks.

\section{Stability analysis for scalar equations}

\subsection{Analytical stability region}

Without loss of generality, we fix the delay $\tau$ to 1 and consider the model equation

$$
y^{\prime}(t)=\alpha y(t)+\beta y(t-1)+\gamma \int_{-1}^{0} y(t+\nu) \mathrm{d} \nu, \quad t>0,
$$

where $\alpha, \beta, \gamma \in \mathbb{R}$ and $y(t)=\psi(t)$ on $[-1,0]$. By looking for solutions of the form $y(t)=\exp (\lambda t)$, we are led to the characteristic equation

$$
\lambda=\alpha+\beta \exp (-\lambda)+\gamma \int_{-1}^{0} \exp (\lambda \nu) \mathrm{d} \nu
$$

In correspondence with the infinite-dimensional nature of equation (2.1), equation (2.2) normally has an infinite number of roots $\lambda \in \mathbb{C}$. Yet, the number of roots in any right half-plane $\operatorname{Re} \lambda>\eta$, for $\eta \in \mathbb{R}$, is finite (cf. Ref. $[16])$. The condition of asymptotic stability of the zero solution to (2.1) is equivalent to the condition that all the roots of algebraic equation (2.2) have a negative real part. The analytical stability region $S_{*}$ is given by

$$
S_{*}=\{(\alpha, \beta, \gamma) \text { : all roots of }(2.2) \text { satisfy } \operatorname{Re} \lambda<0\} .
$$

We use the notation $S_{*}(\beta)$ to denote the stability region in the $(\alpha, \gamma)$-plane for a fixed $\beta$. We proved in Ref. [19] that $S_{*}(\beta)$ is bounded above by the line

$$
C_{*}(\beta)=\{(\alpha, \gamma): \alpha+\gamma=-\beta\}
$$

and bounded below by the curve

$$
\begin{aligned}
C_{0}(\beta)=\{(\alpha, \gamma): & \alpha(\theta)=\beta+\frac{\theta \sin \theta}{1-\cos \theta}, \\
\gamma(\theta) & \left.=\frac{1}{2}\left(\beta^{2}-\alpha^{2}(\theta)-\theta^{2}\right), \theta \in(0,2 \pi)\right\} .
\end{aligned}
$$

As an illustration, we have drawn the stability regions in the $(\alpha, \gamma)$-plane for two different values of $\beta$ in Fig. 1 .

\subsection{Runge-Kutta-Pouzet methods for delay integro-differential equations}

Let $(A, b, c)$ denote a given Runge-Kutta method characterized by the $s \times s$ matrix $A=\left(a_{i j}\right)$ and vectors $b=\left(b_{1}, \ldots, b_{s}\right)^{\mathrm{T}}, c=\left(c_{1}, \ldots, c_{s}\right)^{\mathrm{T}}$. In this 


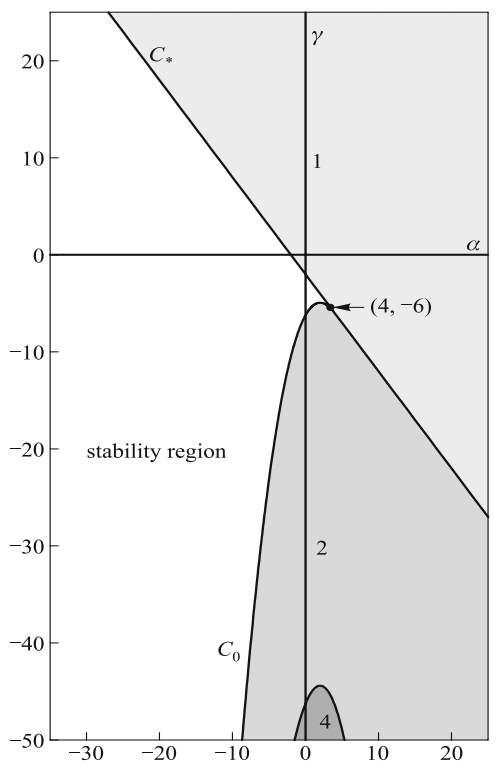

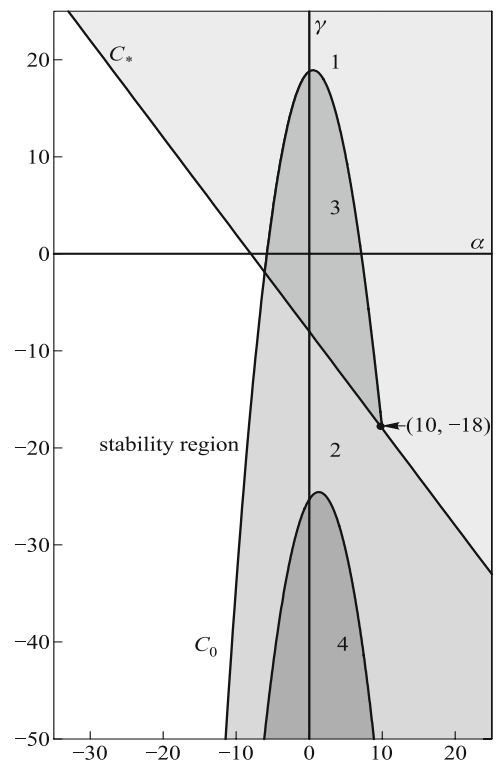

(b)

Fig. 1 Stability region of (2.1), bounded by line $C_{*}(\beta)$ and by curve $C_{0}(\beta)$ in $(\alpha, \gamma)$-plane ((a) $\beta=2$; (b) $\beta=8)$ together with curves separating regions with different numbers (as indicated) of roots in the right half-plane

paper we assume that

$$
\sum_{j=1}^{s} b_{j}=1
$$

Set $t_{n}=n h, n \in \mathbb{Z}$, with $h=1 / m$ for $m$ a positive integer. Approximating both differential and integral parts in (2.1) with the Runge-Kutta method, we find the following scheme (cf. Ref. [22]):

$$
\left\{\begin{array}{l}
Y_{i}^{(n)}=y_{n}+h \sum_{j=1}^{s} a_{i j}\left(\alpha Y_{j}^{(n)}+\beta Y_{j}^{(n-m)}+\gamma G_{j}^{(n)}\right), \quad i=1, \ldots, s, \\
G_{i}^{(n)}=h \sum_{j=1}^{s} a_{i j} Y_{j}^{(n)}+h \sum_{k=1}^{m} \sum_{j=1}^{s} b_{j} Y_{j}^{(n-k)}-h \sum_{j=1}^{s} a_{i j} Y_{j}^{(n-m)}, \\
y_{n+1}=y_{n}+h \sum_{j=1}^{s} b_{j}\left(\alpha Y_{j}^{(n)}+\beta Y_{j}^{(n-m)}+\gamma G_{j}^{(n)}\right),
\end{array}\right.
$$

where $y_{n}$ and $Y_{j}^{(n)}$ are approximations to $y\left(t_{n}\right)$ and $y\left(t_{n}+c_{j} h\right)$, respectively, and $G_{i}^{(n)}$ denotes an approximation to the integral

$\int_{-1}^{0} y\left(t_{n}+c_{i} h+\nu\right) \mathrm{d} \nu=\int_{t_{n}}^{t_{n}+c_{i} h} y(\nu) \mathrm{d} \nu+\int_{t_{n-m}}^{t_{n}} y(\nu) \mathrm{d} \nu-\int_{t_{n-m}}^{t_{n-m}+c_{i} h} y(\nu) \mathrm{d} \nu$. 
Koto [22] has pointed out that this scheme has the same order as the underlying Runge-Kutta method for ordinary differential equations. This follows from the Pouzet-type Runge-Kutta theory for Volterra integro-differential equations by Brunner, Hairer and Norsett [5] and Lubich [24]. Note that, here, we do not consider the computation of the required initial values.

The characteristic equation of scheme (2.4) is given by

$$
\operatorname{det} M(m, \xi)=0 \text {. }
$$

With $I$ standing for the $s \times s$ identity matrix and $e=(1, \ldots, 1)^{\mathrm{T}} \in \mathbb{R}^{s}$, we have

$$
\begin{aligned}
& M(m, \xi) \\
& =\left[\begin{array}{cc}
I-\frac{\alpha+\beta \xi^{-m}}{m} A-\frac{\gamma\left(1-\xi^{-m}\right)}{m^{2}} A^{2}-\frac{\gamma}{m^{2}} \sum_{k=1}^{m} \xi^{-k} A e b^{\mathrm{T}} & -e \\
-\frac{\alpha+\beta \xi^{-m}}{m} b^{\mathrm{T}}-\frac{\gamma\left(1-\xi^{-m}\right)}{m^{2}} b^{\mathrm{T}} A-\frac{\gamma}{m^{2}} \sum_{k=1}^{m} \xi^{-k} b^{\mathrm{T}} & \xi-1
\end{array}\right] .
\end{aligned}
$$

The numerical stability region $S_{m}$ of the scheme for any fixed $m$ is given by

$$
S_{m}=\{(\alpha, \beta, \gamma): \text { all roots of }(2.5) \text { satisfy }|\xi|<1\} .
$$

For any fixed $\beta$, we will use $S_{m}(\beta)$ to denote the stability region in the $(\alpha, \gamma)$-plane.

\subsection{Two examples}

First, we consider $\Theta$-methods, which are Runge Kutta methods with $(A, b, c)$ $=(\Theta, 1, \Theta)$. In this case, $M(m, \xi)$ will be denoted by $M_{\Theta}(m, \xi)$ and satisfies

$$
M_{\Theta}(m, \xi)=\left[\begin{array}{cc}
1-\frac{\left(\alpha+\beta \xi^{-m}\right) \Theta}{m}-\frac{\gamma\left(1-\xi^{-m}\right) \Theta^{2}}{m^{2}}-\frac{\Theta \gamma}{m^{2}} \sum_{k=1}^{m} \xi^{-k} & -1 \\
-\frac{\alpha+\beta \xi^{-m}}{m}-\frac{\gamma\left(1-\xi^{-m}\right) \Theta}{m^{2}}-\frac{\gamma}{m^{2}} \sum_{k=1}^{m} \xi^{-k} & \xi-1
\end{array}\right]
$$

It is known that all $\Theta$-methods with $\Theta \in[1 / 2,1]$ can preserve the stability of the analytical solution $(\tau(0)$-stability, cf. Ref. [11]) in the case of fixed delay problems (i.e., $\gamma=0$ ). Here we show that this does not necessarily hold when $\gamma \neq 0$.

Proposition 2.1 If $\Theta>5 / 6$, then $S_{*} \nsubseteq S_{2}$.

Proof We fix $\beta=2$ and prove that $S_{*}(2) \nsubseteq S_{2}(2)$. Stability region $S_{*}(2)$ is depicted in Fig. 1(a). $S_{*}(2)$ is bounded to the right by the line $C_{*}(2): \alpha+\gamma=$ $-2(\alpha<4)$ and the curve $C_{0}(2)$, which intersect each other only at the point $(\alpha(0), \gamma(0))=(4,-6)$.

It is easy to verify that $\operatorname{det} M_{\Theta}(m,-1)=0$ if and only if

$$
(2 \Theta-1)\left(\alpha+(-1)^{m} \beta+\frac{1}{2 m} \gamma\left(1-(-1)^{m}\right)(2 \Theta-1)\right)=2 m
$$


Hence, when $m=2$, the line

$$
\alpha=\frac{4}{2 \Theta-1}-2
$$

lies outside $S_{2}(2)$. From the assumption $\Theta>5 / 6$ it follows that this line lies in the left half-plane $\alpha<4$, i.e., this line will cross the analytical stability region $S_{*}(2)$. Hence, $S_{*}(2) \nsubseteq S_{2}(2)$, which further gives $S_{*} \nsubseteq S_{2}$. This completes the proof.

Remark 2.2 In fact, our numerical investigation seems to indicate that

$$
S_{*} \subseteq \bigcap_{m=1}^{\infty} S_{m}
$$

possibly only for $\Theta=1 / 2$ although

$$
S_{*}(0) \subseteq \bigcap_{m=1}^{\infty} S_{m}(0)
$$

possibly for all $\Theta \in[1 / 2,1]$ (see, for example, Fig. 2).

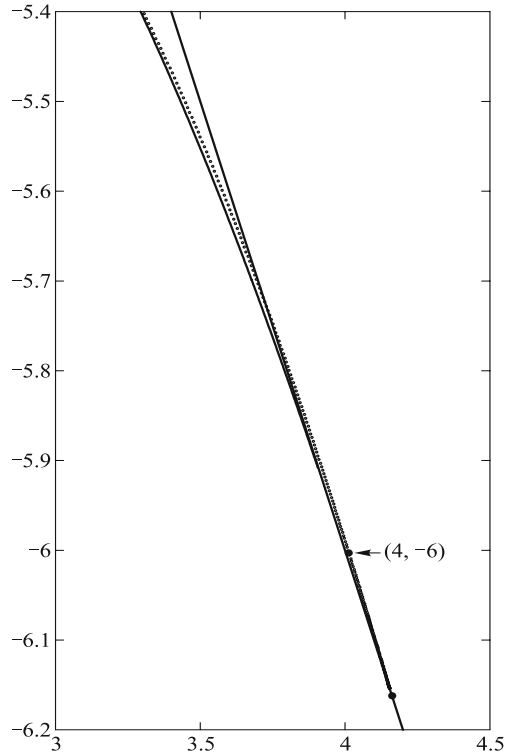

(a)

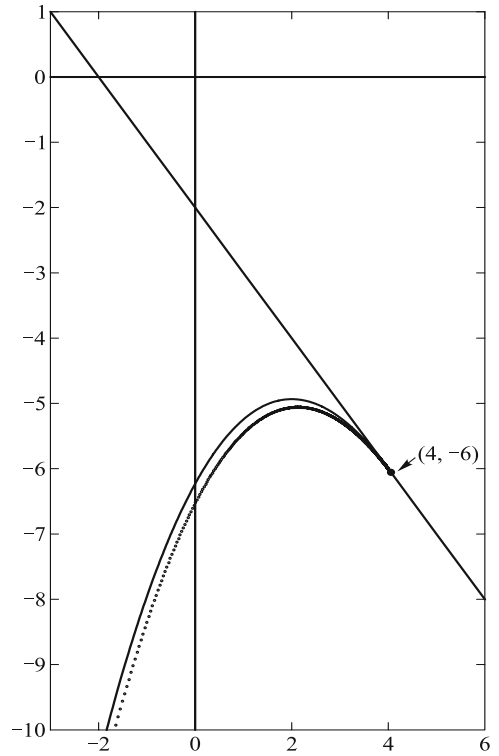

(b)

Fig. 2 Local boundaries of analytical stability region and numerical stability region of $\Theta$-methods for $\beta=2$ and $m=8$. (a) Case $\Theta=0.6$; the analytical stability region is the area between the two solid lines, starting at $(4,-6)$. The numerical boundary locus (dotted line), starting near $(4.15,-6.15)$, crosses the analytical region. (b) Case $\Theta=0.5$; the numerical boundary locus (dotted) lies below $C_{0}(2)$ starting at $(4,-6)$ 
Next, we provide an example of an $A$-stable method that violates $S_{*}(0) \subseteq$ $\bigcap_{m=1}^{\infty} S_{m}(0)$. To that end, we consider the 2 -stage Lobatto IIIC method

\begin{tabular}{c|cc}
0 & $1 / 2$ & $-1 / 2$ \\
1 & $1 / 2$ & $1 / 2$ \\
\hline & $1 / 2$ & $1 / 2$
\end{tabular}

Using the boundary locus technique (cf. Refs. [1,2]), we draw a part of the boundary of the stability region in the $(\alpha, \gamma)$-plane for $\beta=0$ in Fig. $3(\mathrm{a})$. One observes that the boundary of the stability region of the method intersects the boundary of the analytical stability region.

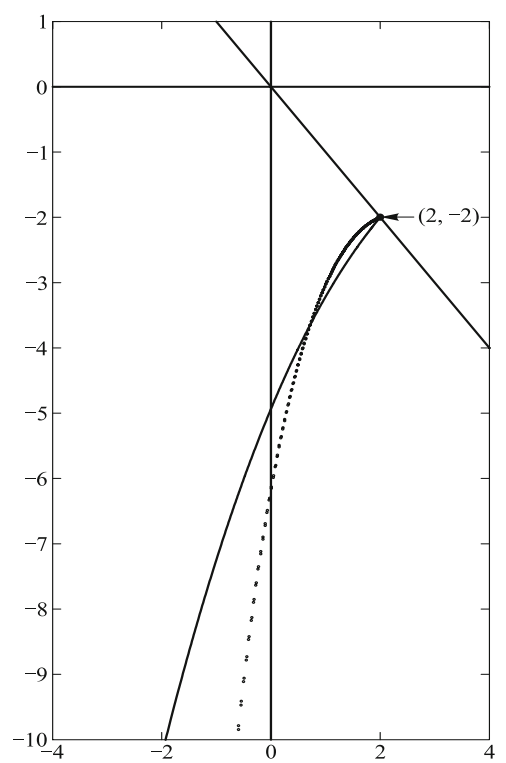

(a)

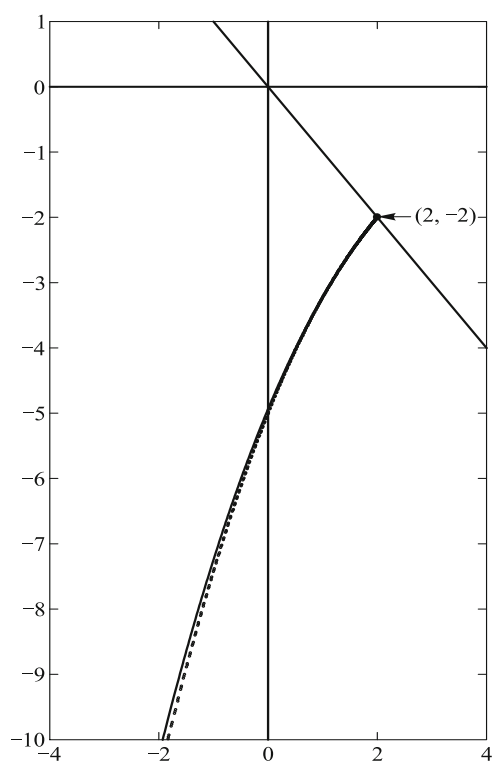

(b)

Fig. 3 Local boundaries of analytical stability region (solid) and numerical stability region (dotted) for $\beta=0$. (a) The 2-stage Lobatto IIIC method for $m=2$;

(b) the 3-stage Gauss method for $m=1$

Remark 2.3 For the case of a fixed delay (i.e., $\beta \neq 0$ and $\gamma=0$ ), it is well known that the Lobatto IIIC methods cannot completely preserve the asymptotic stability of the analytical solution (cf. Refs. [10,13]). Here, we showed that a similar result holds for the distributed delay case $\beta=0$ and $\gamma \neq 0$.

\subsection{Gauss methods}

In this subsection, we derive a positive result on the stability of Runge-KuttaPouzet methods of Gauss type. Since the roots $\xi$ of (2.5) depend continuously 
on the parameters $\alpha, \beta$ and $\gamma$, we conclude that

$$
S_{*} \subseteq \bigcap_{m=1}^{\infty} S_{m}
$$

if, for every $m \geqslant 1$, all the values $(\alpha, \beta, \gamma)$ satisfying (2.5) with $|\xi|=1$ lie outside the analytical stability region $S_{*}$. This property is central to the so-called boundary locus or root locus technique (cf. Refs. [1,2]). For our analysis, we introduce the following notations:

$$
\begin{gathered}
U_{m}=\{(\alpha, \beta, \gamma):(2.5) \text { has at least a root } \xi \text { with }|\xi|=1\}, \\
U_{m}^{1}=\{(\alpha, \beta, \gamma):(2.5) \text { has at least a root } \xi \text { with } \xi=1\}, \\
U_{m}^{2}=\{(\alpha, \beta, \gamma):(2.5) \text { has at least a root } \xi \text { with }|\xi|=1 \text { but } \xi \neq 1\} .
\end{gathered}
$$

The notations $U_{m}(\beta), U_{m}^{1}(\beta)$ and $U_{m}^{2}(\beta)$ stand for the corresponding sets in the $(\alpha, \gamma)$-plane for any fixed $\beta$. Note that

$$
\partial S_{m} \subseteq U_{m}=U_{m}^{1} \cup U_{m}^{2}
$$

We remark that $U_{m}^{1}$ and $U_{m}^{2}$ may intersect each other. In that case, (2.5) has at least two roots with $|\xi|=1$ and one of them is $\xi=1$.

Lemma 2.4 For any Runge-Kutta method (2.4) and $m \geqslant 1$,

$$
C_{*} \subseteq U_{m}^{1} \subseteq U_{m}
$$

Proof A straightforward computation shows

$$
M(m, 1)=\left[\begin{array}{cc}
I-\frac{\alpha+\beta}{m} A-\frac{\gamma}{m} A e b^{\mathrm{T}} & -e \\
-\frac{\alpha+\beta+\gamma}{m} b^{\mathrm{T}} & 0
\end{array}\right] .
$$

Therefore, $\operatorname{det} M(m, 1)=0$ if $\alpha+\beta+\gamma=0$. This completes the proof.

In general, we do not necessarily have $C_{*}=U_{m}^{1}$. For example, $U_{m}^{1}$ also contains the line $\alpha+\beta=2 m$ for the 2-stage Lobatto IIIC method (2.6).

Lemma 2.5 For any Gauss method (2.4) and $m \geqslant 1$,

$$
C_{*}=U_{m}^{1}
$$

Proof Our proof is based on the so-called $W$-transformation. According to Th 5.6 of Chap. IV in Ref. [15], there exists for each Gauss method a matrix $W$ such that

$$
W^{-1} A W=\left[\begin{array}{ccccc}
1 / 2 & -\xi_{1} & & & \\
\xi_{1} & 0 & -\xi_{2} & & \\
& \xi_{2} & \ddots & \ddots & \\
& & \ddots & 0 & -\xi_{s-1} \\
& & & \xi_{s-1} & 0
\end{array}\right]=: X_{G}
$$


with $\xi_{k}=1 /\left(2 \sqrt{4 k^{2}-1}\right)$. This matrix also satisfies

$$
W^{\mathrm{T}} B=W^{-1}, \quad W^{\mathrm{T}} b=e_{1},
$$

where

$$
B=\operatorname{diag}\left(b_{1}, \ldots, b_{s}\right), \quad e_{1}=(1,0, \ldots, 0)^{\mathrm{T}} .
$$

Hence,

$$
\left[\begin{array}{cc}
W^{-1} & 0 \\
0 & 1
\end{array}\right] M(m, 1)\left[\begin{array}{cc}
W & 0 \\
0 & 1
\end{array}\right]=\left[\begin{array}{cc}
I-\frac{\alpha+\beta}{m} X_{G}-\frac{\gamma}{m} X_{G} e_{1} e_{1}^{\mathrm{T}} & -e_{1} \\
-\frac{\alpha+\beta+\gamma}{m} e_{1}^{\mathrm{T}} & 0
\end{array}\right] .
$$

Expanding the determinant of the right-hand side along the last column, we find

$$
\operatorname{det} M(m, 1)=-\frac{\alpha+\beta+\gamma}{m} \operatorname{det} \Xi
$$

where

$$
\Xi=\left[\begin{array}{cccc}
1 & \frac{\xi_{2}(\alpha+\beta)}{m} & & \\
-\frac{\xi_{2}(\alpha+\beta)}{m} & \ddots & \ddots & \\
& \ddots & 1 & \frac{\xi_{s-1}(\alpha+\beta)}{m} \\
& & -\frac{\xi_{s-1}(\alpha+\beta)}{m} & 1
\end{array}\right]
$$

Therefore,

$$
\operatorname{det} M(m, 1)=0 \Longleftrightarrow \alpha+\beta+\gamma=0,
$$

which gives $U_{m}^{1}=C_{*}$.

In order to determine $U_{m}^{2}$, we present some preliminary results first.

Lemma 2.6 For $\xi \neq 1$, the characteristic equation (2.5) is equivalent to

$$
\begin{aligned}
& \operatorname{det}\left[I-\frac{\alpha+\beta \xi^{-m}}{m}\left(A+\frac{1}{\xi-1} e b^{\mathrm{T}}\right)-\frac{\gamma\left(1-\xi^{-m}\right)}{m^{2}}\left(A+\frac{1}{\xi-1} e b^{\mathrm{T}}\right)^{2}\right] \\
& \quad=0 .
\end{aligned}
$$

Proof The conclusion follows from the relation

$$
\begin{aligned}
& {\left[\begin{array}{cc}
I & \frac{1}{\xi-1} e \\
0 & 1
\end{array}\right] M(m, \xi)} \\
& =\left[\begin{array}{cc}
I-\frac{\alpha+\beta \xi^{-m}}{m}\left(A+\frac{1}{\xi-1} e b^{\mathrm{T}}\right)-\frac{\gamma\left(1-\xi^{-m}\right)}{m^{2}}\left(A+\frac{1}{\xi-1} e b^{\mathrm{T}}\right)^{2} & 0 \\
-\frac{\alpha+\beta \xi^{-m}}{m} b^{\mathrm{T}}-\frac{\gamma\left(1-\xi^{-m}\right)}{m^{2}} b^{\mathrm{T}}\left(A+\frac{1}{\xi-1} e b^{\mathrm{T}}\right) & \xi-1
\end{array}\right] .
\end{aligned}
$$


Remark 2.7 The proof actually implies that $\operatorname{det} M(m, \xi)$ is equal to

$$
(\xi-1) \operatorname{det}\left[I-\frac{\alpha+\beta \xi^{-m}}{m}\left(A+\frac{1}{\xi-1} e b^{\mathrm{T}}\right)-\frac{\gamma\left(1-\xi^{-m}\right)}{m^{2}}\left(A+\frac{1}{\xi-1} e b^{\mathrm{T}}\right)^{2}\right] .
$$

Hence, $\xi=1$ is a removable singularity of $\operatorname{det} M(m, \xi)$ because the matrix entries of $M(m, \xi)$ are continuous functions of $\xi$, hence also $\operatorname{det} M(m, \xi)$ is continuous with respect to $\xi$.

Let

$$
R(z)=1+b^{\mathrm{T}} z(I-A z)^{-1} e
$$

be the stability function of a Runge-Kutta method (cf. Ref. [15]). The lemma below is based on the following characterization of $R(z)$ :

$$
R(z)=\frac{\operatorname{det}\left(I-z A+z e b^{\mathrm{T}}\right)}{\operatorname{det}(I-z A)} .
$$

Lemma 2.8 The following equality holds:

$$
\begin{aligned}
& (\xi-1) \operatorname{det}\left(I-z\left(A+\frac{1}{\xi-1} e b^{\mathrm{T}}\right)\right) \\
& \quad=\xi \operatorname{det}(I-z A)-\operatorname{det}\left(I-z A+z e b^{\mathrm{T}}\right),
\end{aligned}
$$

where the left-hand side is interpreted as the limit for $\xi \rightarrow 1$ when $\xi=1$. Furthermore, if and only if the polynomials $\operatorname{det}(I-z A)$ and $\operatorname{det}\left(I-z A+z e b^{\mathrm{T}}\right)$ have no common root, the following equivalence holds:

$$
(\xi-1) \operatorname{det}\left[I-z\left(A+\frac{1}{\xi-1} e b^{T}\right)\right]=0 \Longleftrightarrow \xi=R(z) .
$$

Proof The conclusion follows from (2.9) and the equality

$$
\begin{aligned}
& {\left[\begin{array}{cc}
I & -\frac{1}{\xi-1} e \\
0 & 1
\end{array}\right]\left[\begin{array}{cc}
I-z\left(A+\frac{1}{\xi-1} e b^{\mathrm{T}}\right) & 0 \\
-z b^{\mathrm{T}} & \xi-1
\end{array}\right]} \\
& =\left[\begin{array}{cc}
I-z A & 0 \\
-z b^{\mathrm{T}} & \xi-R(z)
\end{array}\right]\left[\begin{array}{cc}
I & -(I-z A)^{-1} e \\
0 & 1
\end{array}\right] .
\end{aligned}
$$

Remark 2.9 Lemma 2.8 shows a relation between the eigenvalues of matrix $A+\frac{1}{\xi-1} e b^{\mathrm{T}}$ and the boundary of the stability region of the method for ODEs if we set $|\xi|=1$. For reducible methods, however, this equivalence no longer holds. For example, consider the following method:

$$
\begin{array}{c|cc}
1 / 2 & 1 / 2 & 0 \\
c_{*} & 0 & c_{*} \\
\hline & 1 & 0
\end{array}
$$


where $c_{*}$ is a pending constant. It is easy to verify that

$$
c_{*} \in \sigma\left(A+\frac{1}{\xi-1} e b^{T}\right), \quad \xi \neq 1
$$

On the other hand, the method can reduce to the implicit midpoint rule, which has the whole imaginary axis as its boundary of the stability region. So equivalence (2.11) does not hold if we set $c_{*}$ as a non-zero constant.

Using order stars, Guglielmi and Hairer [13] proved the following result which will play an important role in our stability analysis.

Lemma 2.10 Let $R(z)$ be symmetric and assume the order star $A$ has the whole imaginary axis as boundary with A lying to the left. Let function $\varphi(y)$ be the argument of $R(\mathrm{i} y)$, i.e.,

$$
R(\mathrm{i} y)=\exp (\mathrm{i} \varphi(y))
$$

in such a way that $\varphi(0)=0$ and $\varphi(y)$ is continuous. Then, the function $\varphi(y)$ is strictly monotonically increasing and it satisfies the following properties:

$$
\varphi(-y)=-\varphi(y), \quad \varphi(y)<y(y>0), \quad \lim _{y \rightarrow \infty} \varphi(y)=s \pi
$$

where $s$ is the number of poles of $R(z)$.

Let $\xi=\exp (\mathrm{i} \varphi), \xi \neq 1$. From Lemma 2.8, we conclude for the Gauss methods that all the eigenvalues of $A+\frac{1}{\xi-1} e b^{\mathrm{T}}$ lie on the imaginary axis. We denote them as $1 /(\mathrm{i} y)$, and using (2.12), we find that equation (2.8) is equivalent to

$$
1+\frac{\mathrm{i}}{m y}(\alpha+\beta \exp (-\mathrm{i} m \varphi))+\frac{\gamma}{m^{2} y^{2}}(1-\exp (-\mathrm{i} m \varphi))=0 .
$$

Separating real and imaginary parts yields

$$
\left\{\begin{array}{l}
1+\frac{1}{m y} \beta \sin m \varphi+\frac{\gamma}{m^{2} y^{2}}(1-\cos m \varphi)=0, \\
\frac{1}{m y}(\alpha+\beta \cos m \varphi)+\frac{\gamma}{m^{2} y^{2}} \sin m \varphi=0 .
\end{array}\right.
$$

These two equalities determine the parameter sets $(\alpha, \beta, \gamma)$ for which the characteristic equation has roots of modulus one. For a given $\beta$, this leads to a set of curves in the $(\alpha, \gamma)$-plane, parameterized by $\varphi$ for $\varphi \in(0, s \pi)$ but $\cos m \varphi \neq 1$. We will denote the curves by $\bar{\alpha}(\varphi)$ and $\bar{\gamma}(\varphi)$, in order to avoid confusion with the curves $\alpha(\theta)$ and $\gamma(\theta)$ defined in $(2.3)$. The set $U_{m}^{2}(\beta)$ is given by

$$
U_{m}^{2}(\beta)=\bigcup_{k=0}^{\lfloor(m s-1) / 2\rfloor} \bar{C}_{k}(\beta)
$$


where $\lfloor x\rfloor$ stands for the integer part of $x$, and

$$
\begin{gathered}
\bar{C}_{k}(\beta)=\left\{(\bar{\alpha}(\varphi), \bar{\gamma}(\varphi)): \varphi \in\left(\frac{2 k \pi}{m}, \frac{2(k+1) \pi}{m}\right)\right\}, \\
\bar{\alpha}(\varphi)=\beta+\frac{m y \sin m \varphi}{1-\cos m \varphi}, \quad \bar{\gamma}(\varphi)=\frac{1}{2}\left(\beta^{2}-\bar{\alpha}^{2}(\varphi)-m^{2} y^{2}\right),
\end{gathered}
$$

where $y$ and $\varphi$ satisfy (2.12). If $m s$ is odd, we specifically define

$$
\bar{C}_{\lfloor(m s-1) / 2\rfloor}(\beta)=\left\{(\bar{\alpha}(\varphi), \bar{\gamma}(\varphi)): \varphi \in\left(\frac{(m s-1) \pi}{m}, s \pi\right)\right\} .
$$

All the curves $\bar{C}_{k}(\beta)$ lie in the area $\gamma \leqslant\left(\beta^{2}-\alpha^{2}\right) / 2 . \bar{C}_{0}(\beta)$ starts at $(\beta+$ $2,-2(\beta+1))$ and approximates $C_{0}(\beta)$. The other curves $\bar{C}_{k}(\beta)$ start at $\infty$ and end at $\infty$ if $m s$ is even. More precisely, we have the following result.

Lemma 2.11 For $k=1,2, \ldots$,

$$
\lim _{m \varphi \rightarrow 2 k \pi+0} \alpha(\theta) \rightarrow+\infty, \quad \lim _{m \varphi \rightarrow 2 k \pi-0} \alpha(\theta) \rightarrow-\infty, \quad \lim _{m \varphi \rightarrow 2 k \pi} \gamma(\theta) \rightarrow-\infty .
$$

These curves are very similar to those of the continuous case in Fig. 1. We do not plot them here. The only case left, that of $\varphi \rightarrow s \pi$ and $m s$ is odd, is more complicated because $\lim _{\varphi \rightarrow s \pi}(1-\cos m \varphi)=2$ and we have to

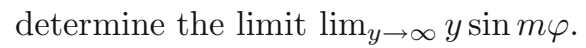

For the $s$-stage Gauss method the stability function is a diagonal Padéapproximation, i.e.,

$$
R_{s s}(z)=\frac{P_{s s}(z)}{Q_{s s}(z)}
$$

with $Q_{s s}(z)=P_{s s}(-z)$ and

$$
P_{s s}(z)=1+\frac{s}{2 s} z+\frac{s(s-1)}{2 s(2 s-1)} \frac{z^{2}}{2 !}+\cdots+\frac{s(s-1) \cdots 1}{2 s \cdots(s+1)} \frac{z^{s}}{s !} .
$$

Lemma 2.12 For the s-stage diagonal Padé-approximation, it holds that

$$
\lim _{y \rightarrow \infty} y \sin \varphi(y)=(-1)^{s-1} 2 s(s+1),
$$

where $y$ and $\varphi$ are defined by (2.12).

Proof A straightforward but technical computation shows that

$$
\lim _{y \rightarrow \infty} y^{2} \frac{R_{s s}^{\prime}(\mathrm{i} y)}{R_{s s}(\mathrm{i} y)}=2 s(s+1),
$$

which, combined with $\lim _{y \rightarrow \infty} \varphi(y)=s \pi$ (Lemma 2.10), gives

$$
\lim _{y \rightarrow \infty} \frac{\sin \varphi(y)}{y^{-1}}=\lim _{y \rightarrow \infty}-\frac{\varphi^{\prime}(y) \cos \varphi(y)}{y^{-2}}=(-1)^{s-1} 2 s(s+1) .
$$


Using Lemma 2.12, we get the following limits for $m s$ odd:

$$
\left\{\begin{aligned}
\lim _{\varphi \rightarrow s \pi} \bar{\alpha}(\varphi) & =\beta+\lim _{\varphi \rightarrow s \pi}(y \sin \varphi) \frac{m \sin m \varphi}{\sin \varphi(1-\cos m \varphi)} \\
& =\beta+s(s+1) m^{2} \\
\lim _{\varphi \rightarrow s \pi} \bar{\gamma}(\varphi) & =-\infty
\end{aligned}\right.
$$

Now we give some further properties of the curves $\bar{C}_{k}(\beta)$.

Lemma 2.13 The curves $\bar{C}_{k}(\beta)$ do not intersect.

Proof Suppose that there exist $\varphi_{1}, \varphi_{2} \in(0, s \pi)$ and corresponding $y_{1}, y_{2}$ defined by $(2.12)$, such that

$$
\bar{\alpha}\left(\varphi_{1}\right)=\bar{\alpha}\left(\varphi_{2}\right), \quad \bar{\gamma}\left(\varphi_{1}\right)=\bar{\gamma}\left(\varphi_{2}\right)
$$

These equalities immediately lead to $y_{1}=y_{2}$. With Lemma 2.10, this gives $\varphi_{1}=\varphi_{2}$, which completes the proof.

Lemma 2.14 The curves $\bar{C}_{k}(\beta)$ are ordered according to $k$.

Proof We consider the intersection of the curves $\bar{C}_{k}(\beta)$ and the line $\alpha=\beta$. The equality $\bar{\alpha}\left(\varphi_{k}\right)=\beta, m \varphi_{k} \in(2 k \pi, 2(k+1) \pi), k=0, \ldots,\left\lfloor\frac{m s-1}{2}\right\rfloor$, implies

$$
\varphi_{k}=\frac{(2 k+1) \pi}{m}, \quad \gamma\left(\varphi_{k}\right)=\frac{-m^{2} y^{2}\left(\varphi_{k}\right)}{2}
$$

where $y\left(\varphi_{k}\right)$ is determined by (2.12) corresponding to $\varphi_{k}$. From the monotonicity of $y(\varphi)$ by Lemma 2.10 , we see that the curves $\bar{C}_{k}, k=$ $0, \ldots,\left\lfloor\frac{m s-1}{2}\right\rfloor$, are ordered in a natural way if $m s$ is even. When $m s$ is odd, considering Lemma 2.13 and (2.13), we conclude that $\bar{C}_{\left\lfloor\frac{m s-1}{2}\right\rfloor}$ is the lowest curve. This completes the proof.

Theorem 2.15 For all Gauss methods, we have

$$
S_{*} \subset \bigcap_{m=1}^{\infty} S_{m}
$$

Proof For any fixed $\beta$, we consider the reference point $(\alpha, \gamma)=(-|\beta|-1,0)$. It is easy to verify by Lemma 2.8 that, for the reference point, no $\xi$ with $|\xi| \geqslant 1$ is a solution to (2.8). By continuity arguments, the whole connected region containing the reference point belongs to the stability region of the method. (In the next theorem we will show that the connected region actually is the stability region.)

First note that both regions are bounded above by $C_{*}(\beta)=U_{m}^{1}(\beta)$. We prove that the curve $\bar{C}_{0}(\beta)$ lies below or to the right of $C_{0}(\beta)$ for any $\beta$ and $m$. To this end, we first prove $C_{0}(\beta)$ intersects $\bar{C}_{0}(\beta)$ only at $(\alpha(0), \gamma(0))$. Suppose that there exist $\theta, m \varphi \in(0,2 \pi)$ such that $\alpha(\theta)=\bar{\alpha}(\varphi)$ and $\gamma(\theta)=$ 
$\bar{\gamma}(\varphi)$. Then we have $\theta=m y$, which gives $m y \in(0,2 \pi)$. From the monotonicity of function $\sin x /(1-\cos x)$ and the fact that $\varphi(y)<y$ (Lemma 2.10), it follows that

$$
\alpha(\theta)=\beta+\frac{m y \sin m y}{1-\cos m y}<\beta+\frac{m y \sin m \varphi}{1-\cos m \varphi}=\bar{\alpha}(\varphi),
$$

which contradicts the earlier assumption $\alpha(\theta)=\bar{\alpha}(\varphi)$.

Next we prove that $\bar{C}_{0}(\beta)$ lies below $C_{0}(\beta)$ (in the case $m s>1$ ) or to the right of $C_{0}(\beta)$ (in the case $m s=1$ ). In the case of $m s>1$, it is easy to verify that

$$
\alpha(\pi)=\bar{\alpha}\left(\frac{\pi}{m}\right)=\beta, \quad \gamma(\pi)=-\frac{\pi^{2}}{2}>\bar{\gamma}\left(\frac{\pi}{m}\right) .
$$

In the case of $m s=1$, for any $\gamma(\theta)=\bar{\gamma}(\varphi)$, one has

$$
\alpha(\theta)<\beta+2=\bar{\alpha}(\varphi)
$$

because $\bar{C}_{0}(\beta)$ is the line $\{(\beta+2, \gamma): \gamma \in(-\infty,-2(\beta+1))\}$ (cf. Ref. [19]). This completes the proof.

Theorem 2.16 For all Gauss methods, the stability region $S_{m}(\beta)$ is bounded above by the line $C_{*}(\beta)$ and below by the curve $\bar{C}_{0}(\beta)$.

Proof At each point of a curve $\bar{C}_{k}(\beta)$, there exists a pair of critical roots $\exp ( \pm \mathrm{i} \varphi)$ exactly on the unit disk. Now we study to which side of the curves the critical roots move outside the unit disk. Let $\xi=\exp (\mu+\mathrm{i} \varphi)$ and let $1 /(x+\mathrm{i} y)$ be an eigenvalue of matrix $A+\frac{1}{\xi-1} e b^{\mathrm{T}}$. Then the characteristic equation (2.8) is equivalent to

$$
1-\frac{\alpha+\beta \exp (-m(\mu+\mathrm{i} \varphi))}{m(x+\mathrm{i} y)}-\frac{\gamma(1-\exp (-m(\mu+\mathrm{i} \varphi)))}{m(x+\mathrm{i} y)^{2}}=0 .
$$

We denote the left-hand side by $F(\alpha, \gamma, \mu+\mathrm{i} \varphi)$. Define

$$
F_{1}(\alpha, \gamma, \mu, \varphi)=\operatorname{Re} F(\alpha, \gamma, \mu+\mathrm{i} \varphi), \quad F_{2}(\alpha, \gamma, \mu, \varphi)=\operatorname{Im} F(\alpha, \gamma, \mu+\mathrm{i} \varphi) .
$$

Calculating the determinant $\operatorname{det} \mathscr{M}$, where

$$
\mathscr{M}=\left[\begin{array}{ll}
\frac{\partial F_{1}}{\partial \alpha} & \frac{\partial F_{1}}{\partial \gamma} \\
\frac{\partial F_{2}}{\partial \alpha} & \frac{\partial F_{2}}{\partial \gamma}
\end{array}\right]_{\mu=0},
$$

and noting that $\mu=0$ implies $x=0$, we find

$$
\operatorname{det} \mathscr{M}=\frac{1-\cos m \varphi}{-m^{3} y^{3}},
$$

where $y$ and $\varphi$ satisfy (2.12). We have

$$
\operatorname{det} \mathscr{M} \begin{cases}<0, & y>0, \\ >0, & y<0 .\end{cases}
$$


We now apply Proposition 2.13 of Chap. XI in Ref. [9]. If we cross a curve $\bar{C}_{k}(\beta)$ to the left with 'left' defined with respect to the direction of increasing $\varphi$-coordinate for $\varphi>0$, the critical roots $\pm \mathrm{i} \varphi$ enter the right half-plane, i.e., the number of roots $\xi$ with $|\xi|>1$ increases by 2 . On the other hand, from a result in Ref. [13], one has the scheme is not asymptotically stable for any $\alpha \geqslant-\beta, \gamma=0$. By continuity arguments, we may conclude that the area $\alpha+\beta+\gamma \geqslant 0$ does not belong to the stability region. This completes the proof.

Remark 2.17 Specializing Theorems 2.15 and 2.16 to the case $\gamma=0$, one regains the stability result for Gauss methods as obtained by Guglielmi and Hairer in Ref. [13]. Our results also extend the results given by Luzyanina, Engelborghs and Roose [25], who focussed on the RHP-stability of linear multistep methods; i.e., they addressed the question as to whether a numerical stability region contains a wedge region given by (3.8), see Remark 3.4 below. Here we analyze whether a numerical stability region contains the complete delay-dependent stability region.

Remark 2.18 Set

$$
z(t)=\int_{t-\tau}^{t} y(\nu) \mathrm{d} \nu
$$

Then, (2.1) can be transformed into a system of delay differential equations:

$$
\left[\begin{array}{l}
y^{\prime}(t) \\
z^{\prime}(t)
\end{array}\right]=\left[\begin{array}{ll}
\alpha & \gamma \\
1 & 0
\end{array}\right]\left[\begin{array}{l}
y(t) \\
z(t)
\end{array}\right]+\left[\begin{array}{cc}
\beta & 0 \\
-1 & 0
\end{array}\right]\left[\begin{array}{l}
y(t-1) \\
z(t-1)
\end{array}\right] .
$$

Hence, certain stability characteristics of (2.1) can be inferred from the existing stability theory for general multi-dimensional systems of delay equations. That theory, however, is not necessarily sharp for systems of type (2.14); more precise results can be derived by studying (2.1) or the particular 2-dimensional system (2.14) directly. We are not aware, for example, of any positive results on delay dependent stability of numerical methods for general multi-dimensional systems of delay equations that would adequately cover equation (2.14). Some negative results, i.e., showing instability, have been obtained (cf. Refs. [12,27]).

Remark 2.19 In this paper, we only consider the finite delay case. A further topic is to study the stability of Volterra integro-differential equations with infinite delays. For example, the following test problem,

$$
y^{\prime}(t)=\alpha y(t)+\beta y(t-\tau)+\gamma \int_{0}^{t-\tau} y(s) \mathrm{d} s,
$$

is worthy of further study and we will address this issue in future work. We remark that, in the case of $\tau=0$, the analytical and numerical stability of such equations was first analyzed by Brunner and Lambert [6] and by Matthys [28]. 


\section{Stability analysis of the multidimensional case}

\subsection{Stability condition for the analytical solution}

In this subsection, we consider the asymptotic stability of multi-dimensional equations of the form

$$
y^{\prime}(t)=L y(t)+M y(t-\tau)+K \int_{t-\tau}^{t} y(\nu) \mathrm{d} \nu, \quad t>0,
$$

where $L, M, K \in \mathbb{C}^{d \times d}$ and $y(t)=\psi(t)$ on $[-\tau, 0]$. The characteristic equation equals

$$
\operatorname{det}\left[\lambda I_{d}-L-M \exp (-\tau \lambda)-K \int_{-\tau}^{0} \exp (\lambda \nu) \mathrm{d} \nu\right]=0,
$$

where $I_{d}$ is the $d \times d$ identity matrix. The zero solution of (3.1) is asymptotically stable if and only if all the roots $\lambda$ of (3.2) have negative real parts. We are not aware of any explicit conditions which are suitable to describe the complete stability region of (3.1), although some sufficient conditions have been found (cf. Refs.[22,31]). In the following, we will address this question and derive some new stability results.

First, we introduce some notations. $\sigma(\cdot)$ and $\rho(\cdot)$ designate the spectrum and spectral radius of a matrix; the sets $\mathbb{C}^{+}, \mathbb{C}^{-}, \mathbb{C}^{0}$ denote the sets of complex numbers with strictly positive real part, strictly negative real part, and zero real part, respectively. The set denoted as $\mathbb{D}$ is the closed complex unit disk. Finally,

$$
\mathbb{Q}=\left\{z \in \mathbb{C}: \quad z=q \int_{-1}^{0} \exp (\mathrm{i} \theta \nu) \mathrm{d} \nu, \theta \in[-2 \pi, 2 \pi], q \in[0,1]\right\},
$$

whose boundary is shown in Fig. 4.

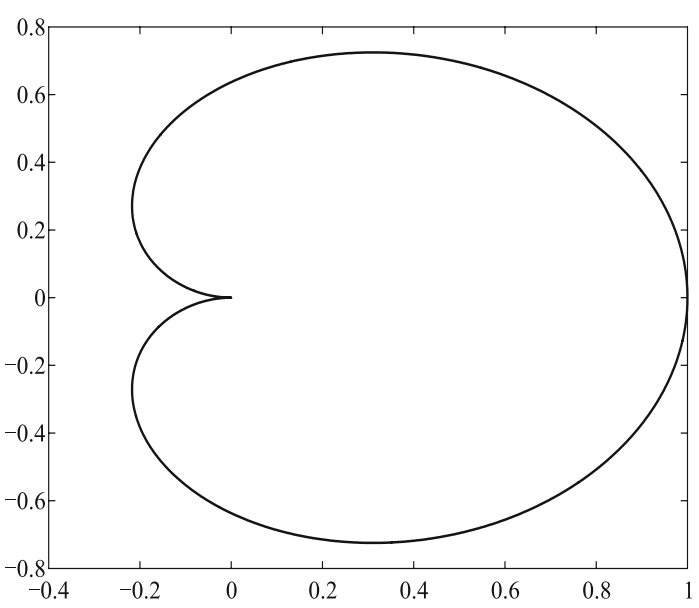

Fig. 4 Boundary of the set $Q$ 


\section{Lemma 3.1}

$$
\left\{\begin{array}{l}
\partial \mathbb{Q}=\left\{z \in \mathbb{C}: z=\int_{-1}^{0} \exp (\mathrm{i} \theta \nu) \mathrm{d} \nu, \theta \in[-2 \pi, 2 \pi]\right\}, \\
\mathbb{Q}=\left\{z \in \mathbb{C}: z=\int_{-1}^{0} \exp (\lambda \nu) \mathrm{d} \nu, \lambda \in \mathbb{C}^{+} \cup \mathbb{C}^{0}\right\} .
\end{array}\right.
$$

Proof Let

$$
z(\theta)=\int_{-1}^{0} \exp (\mathrm{i} \theta \nu) \mathrm{d} \nu, \quad \theta \in[-2 \pi, 2 \pi]
$$

Then

$$
z(2 \pi)=z(-2 \pi)=0, \quad \arg z(\theta)=\frac{\theta}{2}, \quad \theta \in(-2 \pi, 2 \pi) .
$$

The monotonicity of the argument of $z(\theta)$ gives the first statement.

Next, we prove the second statement. Let

$$
F(\lambda)=\int_{-1}^{0} \exp (\lambda \nu) \mathrm{d} \nu
$$

Then $F(\lambda)$ is analytical. It maps the open set $\mathbb{C}^{+}$into a connected open set. Also, it maps the closed right half-plane in the extended complex plane into a closed set, which we denote by $\mathbb{Q}_{0}$. Considering $|F(\lambda)| \leqslant 1$ when $\operatorname{Re} \lambda \geqslant 0$ and $\lim _{\theta \rightarrow \pm \infty} F(\mathrm{i} \theta)=0=F(2 \pi \mathrm{i})$, one can see that $\mathbb{Q}_{0}$ is bounded and

$$
\partial \mathbb{Q}_{0} \subseteq\{z \in \mathbb{C}: z=F(\mathrm{i} \theta), \theta \in \mathbb{R}\} \subseteq \mathbb{Q}_{0} .
$$

Using the first statement of $(3.3)$, we have $\mathbb{Q} \subseteq \mathbb{Q}_{0}$. On the other hand, it is easy to verify that $F(\mathrm{i} \theta) \in \mathbb{Q}$ when $|\theta|>2 \pi$. Hence, $\partial \mathbb{Q}_{0} \subseteq \mathbb{Q}$, which gives $\mathbb{Q}=\mathbb{Q}_{0}$.

Theorem 3.2 System (3.1) is asymptotically stable if

$$
\sigma\left(L+\xi_{1} M+\xi_{2} \tau K\right) \subset \mathbb{C}^{-}, \quad \forall \xi_{1} \in \mathbb{D}, \forall \xi_{2} \in \mathbb{Q},
$$

or, equivalently,

$$
\sigma\left(L+\xi_{1} M+\xi_{2} \tau K\right) \subset \mathbb{C}^{-}, \quad \forall \xi_{1} \in \partial \mathbb{D}, \forall \xi_{2} \in \partial \mathbb{Q} .
$$

Proof Suppose that (3.2) has a root $\lambda \in \mathbb{C}^{+} \cup \mathbb{C}^{0}$. Then

$$
\lambda \in \sigma\left(L+M \exp (-\tau \lambda)+K \int_{-\tau}^{0} \exp (\lambda \nu) \mathrm{d} \nu\right) .
$$

Set

$$
\xi_{1}=\exp (-\tau \lambda), \quad \xi_{2}=\frac{1}{\tau} \int_{-\tau}^{0} \exp (\lambda \nu) \mathrm{d} \nu .
$$

Then $\xi_{1} \in \mathbb{D}$ and $\xi_{2} \in \mathbb{Q}$. This contradicts with (3.4). Finally, (3.5) follows from (3.4) by the properties of analytic functions. 
Remark 3.3 A condition similar to (3.5) has been used to examine the stability of numerical schemes (cf. Ref. [25]) for the scalar equation

$$
y^{\prime}(t)=\alpha y(t)+\gamma \int_{t-\tau}^{t} y(\nu) \mathrm{d} \nu, \quad \alpha, \gamma \in \mathbb{R} .
$$

Remark 3.4 In the case of fixed delay (i.e., $K=0$ ), it is known that condition (3.4) or (3.5) is stronger than the sufficient and necessary condition for delay-independent stability. That is, the stability region given by (3.5) is a subset of the delay-independent stability region.

Remark 3.5 In the case of distributed delay (i.e., $K \neq 0$ ), however, the above observation does not hold. Condition (3.4) is not easily related to the delay-independent stability condition. Consider, for example, equation (3.6). The delay-independent stability region was studied by Koto [22], and is given by

$$
\alpha<0, \quad \frac{-\alpha^{2}}{2}<\gamma \leqslant 0
$$

For this problem, (3.5) reduces to

$$
4.6033 \alpha \approx \frac{-\alpha}{\min _{\theta \in[-2 \pi, 2 \pi]} \frac{\sin \theta}{\theta}}<\tau \gamma<-\alpha .
$$

We have drawn in Fig. 5 the boundaries of these two regions as well as the delay-dependent stability region given by (2.3). The boundary defined by the second inequality of $(3.8)$, is just the line $C_{*}(0)$. The boundary defined by the first inequality appears to be the tangent line to the curve $C_{0}(0)$. This means that (3.8) defines the biggest wedge with top in the origin contained in the delay-dependent stability region.

\subsection{Numerical stability}

Application of a Runge-Kutta method $(A, b, c)$ of Pouzet type with constant stepsize $h=\tau / m$ to (3.1), leads to the following scheme:

$$
\begin{cases}Y_{i}^{(n)}=y_{n}+h \sum_{j=1}^{s} a_{i j}\left(L Y_{j}^{(n)}+M Y_{j}^{(n-m)}+K G_{j}^{(n)}\right), & i=1, \ldots, s, \\ G_{i}^{(n)}=h \sum_{j=1}^{s} a_{i j} Y_{j}^{(n)}+h \sum_{k=1}^{m} \sum_{j=1}^{s} b_{j} Y_{j}^{(n-k)}-h \sum_{j=1}^{s} a_{i j} Y_{j}^{(n-m)}, i=1, \ldots, s, \\ y_{n+1}=y_{n}+h \sum_{j=1}^{s} b_{j}\left(L Y_{j}^{(n)}+M Y_{j}^{(n-m)}+K G_{j}^{(n)}\right) .\end{cases}
$$

The corresponding characteristic equation is of the form

$$
\operatorname{det} \mathbb{M}(m, \xi)=0
$$

Using $\otimes$ to denote the Kronecker product, we have 


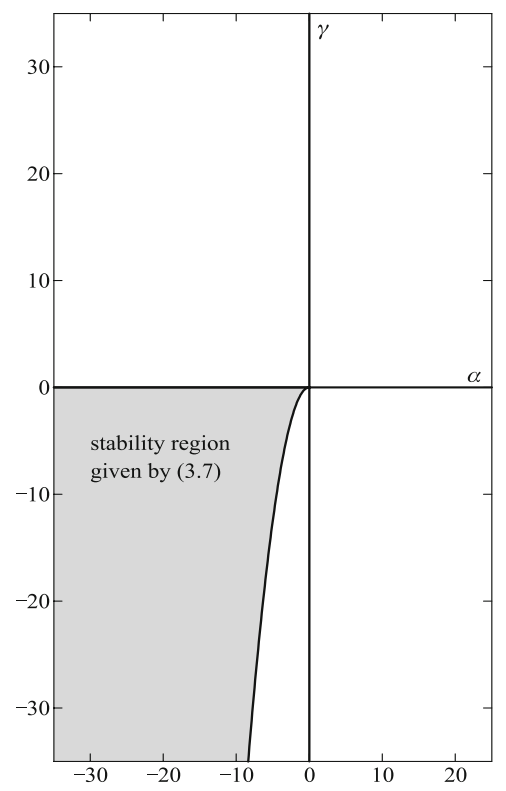

(a)

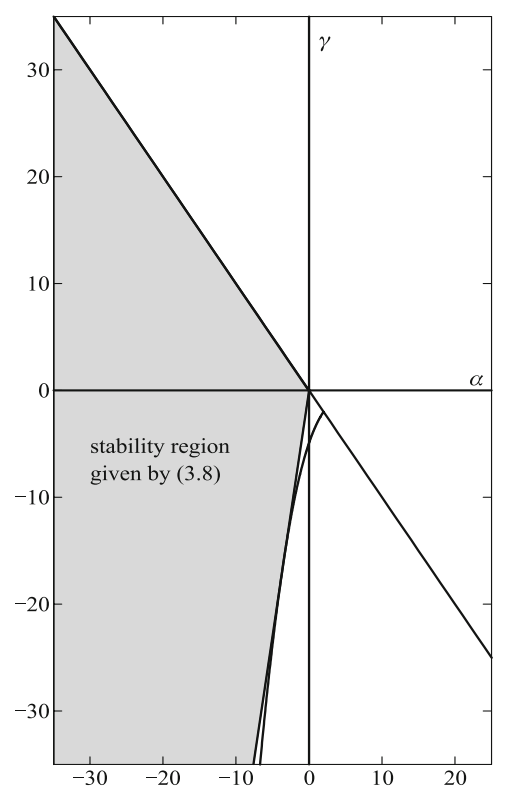

(b)

Fig. 5 (a) Delay-independent stability region of (3.6), given by (3.7); (b) stability region as defined by the sufficient delay-dependent condition (3.8) for $\tau=1$; also shown the boundary $C_{0}(0)$ of the exact delay-dependent stability region defined by $(2.3)$

$$
\begin{aligned}
& \mathbb{M}(m, \xi) \\
& =\left[\begin{array}{cc}
I_{s d}-h A \otimes\left(L+\xi^{-m} M\right)-h^{2}\left(1-\xi^{-m}\right)\left(A\left(A+\frac{1}{\xi-1} e b^{\mathrm{T}}\right)\right) \otimes K & -e \otimes I_{d} \\
-h b^{\mathrm{T}} \otimes\left(L+\xi^{-m} M\right)-h^{2}\left(1-\xi^{-m}\right)\left(b^{\mathrm{T}}\left(A+\frac{1}{\xi-1} e b^{\mathrm{T}}\right)\right) \otimes K & (\xi-1) I_{d}
\end{array}\right] .
\end{aligned}
$$

Koto [22] has proved that all $A$-stable Runge-Kutta-Pouzet methods preserve the delay-independent stability of (3.1). We naturally wonder whether all $A$-stable methods also preserve the analytical stability region given by delay-dependent stability condition (3.4). The following result shows that the answer is negative.

Theorem 3.6 Consider equation (3.6). For any $\gamma>0$ with $\alpha+\tau \gamma<0$, there exists an $A$-stable method $(A, b, c)$ such that (3.9) is unstable for some stepsize $h=\tau / m$.

Proof The proof is constructive. We consider the $\Theta$-method applied to (3.6). In this case, the characteristic polynomial is given by

$$
\operatorname{det} \mathbb{M}_{\Theta}(m, \xi)=\xi-1-\frac{\tau}{m}((\xi-1) \Theta+1)\left(\alpha+\left(1-\xi^{-m}\right) \frac{(\xi-1) \Theta+1}{m(\xi-1)} \gamma \tau\right)
$$

Therefore,

$$
\operatorname{det} \mathbb{M}_{\Theta}(1,2)=1-\tau(\Theta+1)\left(\alpha+\frac{\Theta+1}{2} \gamma \tau\right)
$$


We have $\operatorname{det} \mathbb{M}_{\Theta}(1,2)>0$ when $\Theta=1$, and $\operatorname{det} \mathbb{M}_{\Theta}(1,2)<0$ when $\Theta$ is sufficiently large. Hence, there exists a $\Theta>1$ such that $\operatorname{det} \mathbb{M}_{\Theta}(1,2)=0$. The corresponding $\Theta$-method (3.9) is unstable. On the other hand, it is well known that every $\Theta$-method with $\Theta \geqslant 1 / 2$ is $A$-stable. So we arrive at the conclusion of the theorem.

Another illustrative example is the 2-stage Lobatto IIIC method (2.6). In Section 4, we will give a numerical investigation to show its instability.

Next, we study the characteristic roots of (3.10) in order to identify methods that can preserve the stability region given by (3.4). We first state a result from Ref. [22].

Lemma 3.7 If $\operatorname{det}(L+M+\tau K) \neq 0, \sigma(L+M) \subset \mathbb{C}^{-}, \sigma(A) \subset \mathbb{C}^{+} \cup \mathbb{C}^{0}$, and the Runge-Kutta method $(A, b, c)$ is A-stable, then $\xi=1$ is not a root of $(3.10)$.

A proof similar to that of Lemma 2.6 leads to the following lemma.

Lemma 3.8 For $\xi \neq 1$, the characteristic equation (3.10) is equivalent to

$$
\begin{aligned}
\operatorname{det} & {\left[I_{s d}-h\left(A+\frac{1}{\xi-1} e b^{\mathrm{T}}\right) \otimes\left(L+\xi^{-m} M\right)-h^{2}\left(1-\xi^{-m}\right)\left(A+\frac{1}{\xi-1} e b^{\mathrm{T}}\right)^{2} \otimes K\right] } \\
& =0 .
\end{aligned}
$$

Now we are in the position to state and prove the main result of this section.

Theorem 3.9 Suppose that the Runge-Kutta method $(A, b, c)$ is A-stable and that $\operatorname{det}\left(I_{s}-z A\right)$ and $\operatorname{det}\left(I_{s}-z A+z e b^{\mathrm{T}}\right)$ have no common roots. Assume that $L, M$ and $K$ satisfy (3.4), and that

$$
\sigma\left(\frac{1-\xi^{-m}}{m}\left(A+\frac{1}{\xi-1} e b^{T}\right)\right) \subset \mathbb{Q} \quad \text { whenever }|\xi|=1 .
$$

Then, difference equation (3.9) is asymptotically stable.

Proof We first show that the assumptions of Lemma 3.7 are satisfied. From condition (3.4) it follows that $\operatorname{det}(L+M+\tau K) \neq 0$ and $\sigma(L+M) \subset \mathbb{C}^{-}$. Since the method is $A$-stable, the stability function $R(z)$ is analytic in $\mathbb{C}^{-}$. Considering (2.9) and the assumptions of the theorem, we have $\sigma(A) \subset \mathbb{C}^{+} \cup$ $\mathbb{C}^{0}$. Hence, Lemma 3.7 guarantees that $\xi=1$ is not a root of $(3.10)$.

The next step uses Lemma 3.8. In order to prove stability of (3.9), it remains to show that any $\xi \in \mathbb{C}$ with $|\xi| \geqslant 1$ and $\xi \neq 1$ is not a root of (3.11).

Let $\lambda_{j}(\xi)(j=1, \ldots, s)$ be the eigenvalues of matrix

$$
\frac{1-\xi^{-m}}{m}\left(A+\frac{1}{\xi-1} e b^{\mathrm{T}}\right)
$$

Those eigenvalues are analytic in the extended complex plane, except at the origin; note that $\xi=1$ is a removable singularity. Each $\lambda_{j}(\xi)$ maps the open 
set $\{\xi \in \mathbb{C}:|\xi|>1\}$ into a connected open set $\mathbb{Q}_{j}$, where $\mathbb{Q}_{j}$ is bounded since $\lambda_{j}(\xi)$ is uniformly bounded for $|\xi| \geqslant 1$. Hence,

$$
\partial \mathbb{Q}_{j} \subset\left\{\lambda_{j}(\xi):|\xi|=1\right\} .
$$

From (3.12) it follows that

$$
\lambda_{j}(\xi) \in \mathbb{Q} \quad \text { whenever }|\xi| \geqslant 1 .
$$

Let $\mu_{j}(\xi)$ be the eigenvalue of matrix $\left(A+\frac{1}{\xi-1} e b^{\mathrm{T}}\right)$ that satisfies

$$
\lambda_{j}(\xi)=\frac{1-\xi^{-m}}{m} \mu_{j}(\xi)
$$

In view of Lemma 2.8, we have

$$
\frac{1}{\mu_{j}(\xi)} \in \mathbb{C} \backslash S_{\mathrm{ODE}} \quad \text { whenever }|\xi| \geqslant 1 \text { but } \xi \neq 1,
$$

where

$$
S_{\mathrm{ODE}}=\{z \in \mathbb{C}:|R(z)|<1\} .
$$

From the assumptions of $A$-stability and condition (3.4), it follows that

$$
\sigma\left(L+\xi^{-m} M+\lambda_{j}(\xi) \tau K\right) \subset \mathbb{C}^{-} \subset S_{\mathrm{ODE}} \quad \text { whenever }|\xi| \geqslant 1 .
$$

Hence, for every $j \in\{1, \ldots, s\}$, one has

$$
\frac{1}{\mu_{j}(\xi)} \notin \sigma\left(L+\xi^{-m} M+\lambda_{j}(\xi) \tau K\right) \quad \text { whenever }|\xi| \geqslant 1 \text { but } \xi \neq 1,
$$

showing that $(3.11)$ has no root $\xi$ with $|\xi| \geqslant 1$ and $\xi \neq 1$. This completes the proof.

Theorem 3.10 All the Gauss methods satisfy (3.12) for every $m \geqslant 1$. Hence, they preserve the stability region given by (3.4).

Proof Consider the $s$-stage Gauss method. Let $\xi=\exp (\mathrm{i} \varphi)$. From Lemma 2.8 , we have all the eigenvalues of $A+\frac{1}{\xi-1} e b^{\mathrm{T}}$ lie on the imaginary axis; we denote them as $1 /(\mathrm{i} y)$. Using (2.12), we see that the eigenvalues of matrix

$$
\frac{1-\xi^{-m}}{m}\left(A+\frac{1}{\xi-1} e b^{\mathrm{T}}\right)
$$

are given by $(1-\exp (-\mathrm{i} m \varphi)) /(\mathrm{i} m y)$, where $y \in(-\infty,+\infty), \varphi \in(-s \pi, s \pi)$. Note that $\varphi$ and $y$ satisfy (2.12) and that $|y|>|\varphi|$ by Lemma 2.10. (The above is interpreted as the limit 1 for $\varphi \rightarrow 0$ when $\varphi=0$.) Finally, (3.12) follows by considering that

$$
\frac{1-\exp (-\mathrm{i} m \varphi)}{\mathrm{i} m y}=\frac{\varphi}{y} \int_{-1}^{0} \exp (\mathrm{i} m \varphi \nu) \mathrm{d} \nu \in \mathbb{Q}
$$


This completes the proof.

Remark 3.11 Maset [27] and Guglielmi [12] proved that no Runge-Kutta method can preserve the asymptotic stability of all systems of delay differential equations of high dimensions. However, high order collocation methods are a class of important methods for solving functional differential equations (cf. Refs. [3,4]). A natural question that follows is to what extent such methods can preserve the stability of continuous systems. Here we obtain a positive result that Gauss methods can preserve the stability of a large class of delay integro-differential equations, which provides a firmer theoretical support for their applications to functional differential equations.

\section{Numerical experiments}

We present some numerical examples in order to confirm our theoretical findings. We consider the scalar equation (2.1) and we apply scheme (2.4). We set $m=10$, i.e., $h=0.1$, and we take the initial values as follows:

$$
Y_{i}^{(k)}=\frac{k}{m}, \quad k=1, \ldots, m ; i=1, \ldots, s ;
$$

and $y_{m+1}=1$. Formula $(2.4)$ is then applied starting with $n=m+1$.

First, we consider the stability region $S_{*}$ defined in Section 2.1. For the parameters

$$
\alpha=3.8, \quad \beta=2, \quad \gamma=-5.801,
$$

it is easy to verify that $(\alpha, \beta, \gamma)$ is just inside $S_{*}$, close to the boundary defined by $\alpha+\gamma=-\beta$ (see Fig. 1). The first 500 steps of the solution obtained by the implicit midpoint rule (i.e., the second-order Gauss method) and obtained by the implicit Euler method (the $\Theta$-method with $\Theta=1$ ) are plotted in Fig. 6 . From the picture, it is obvious that the former is asymptotically stable; the latter is unstable. This is in accordance with the theory from Section 2.

Next, we consider the stability region given in Section 3. For

$$
\alpha=-3.6195, \quad \beta=0, \quad \gamma=-16.66, \quad \tau=1 \text {, }
$$

one can verify that condition (3.8) holds (with the left inequality almost being an equality). Hence, $(\alpha, \beta, \gamma)$ is just inside the stability region defined by (3.4). The envelope of the numerical solution obtained with the 2 -stage Gauss method is plotted in Fig. 7(a). (The solution itself oscillates too rapidly to be plotted.) The asymptotic stability of the numerical solution is clearly visible, which illustrates the validity of Theorem 3.10. The envelope of the solution obtained with the 2-stage Lobatto IIIC method (2.6) is shown in Fig. 7(b). The instability of the method is obvious, although the growth of error is at first not so dramatic as with the implicit Euler method in Fig. 6. This result confirms that not all $A$-stable methods preserve the stability region defined by $(3.4)$. 


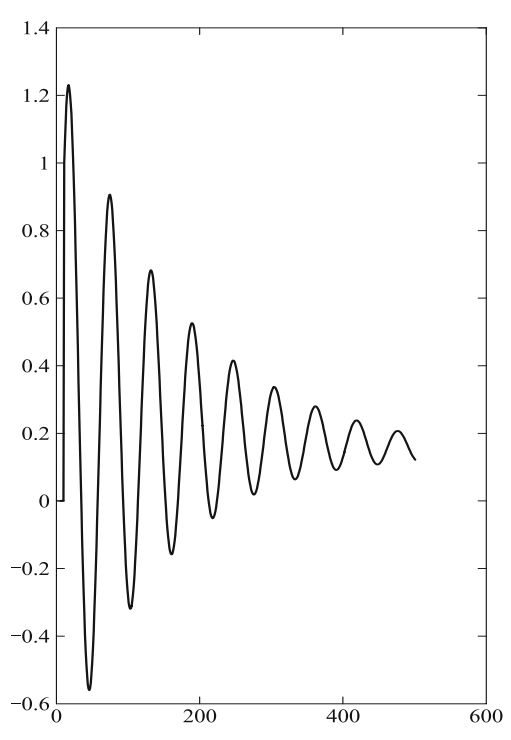

(a)

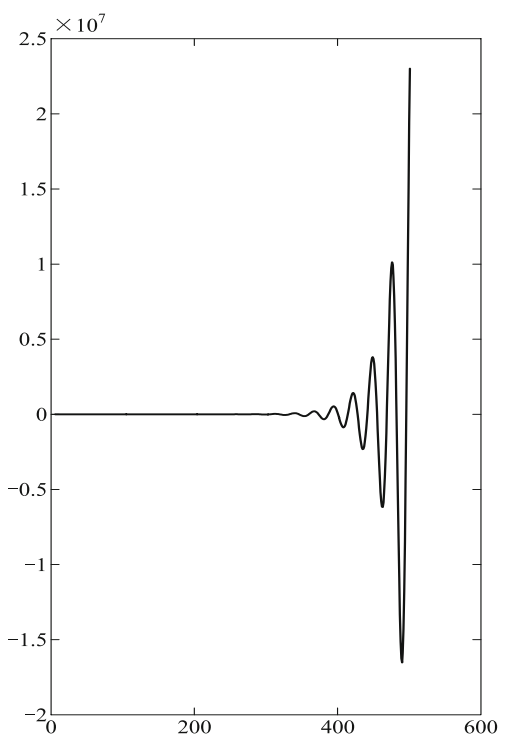

(b)

Fig. 6 Numerical solution obtained with the implicit midpoint rule (a) and numerical solution obtained with the implicit Euler method (b) for $\alpha=3.8, \beta=2, \gamma=-5.801$

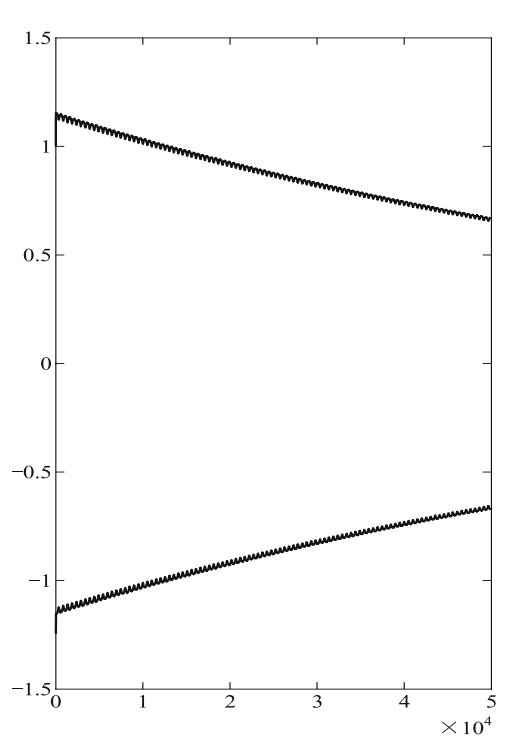

(a)

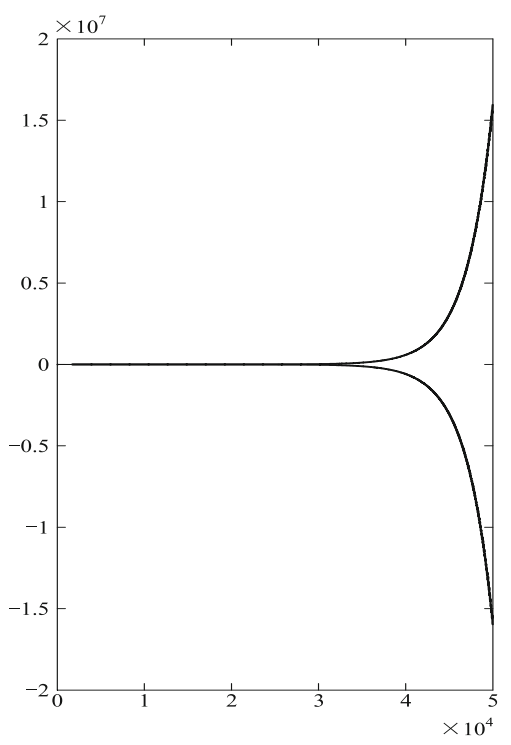

(b)

Fig. 7 Envelope of the numerical solution obtained with the 2-stage Gauss method (a) and envelope of the numerical solution obtained with the 2-stage Lobatto IIIC method

(b) for $\alpha=-3.6195, \beta=0, \gamma=-16.66$ 


\section{Concluding remarks}

We have studied the asymptotic stability of a fairly general class of scalar distributed delay equations and of systems of distributed delay equations. For the scalar case, an exact characterization of the delay-dependent stability region is given. In the system case, a subset of the delay-dependent stability region is identified, which differs from the delay-independent region that was studied elsewhere. We have shown that Gauss-Pouzet type RungeKutta methods preserve the delay-dependent analytical stability region, derived from our sufficient condition for stability of the continuous solution. Preliminary numerical investigations have shown that the Radau methods also satisfy (3.12). Yet, a rigorous proof is still missing. This case requires further investigation.

Acknowledgements The authors wish to thank the anonymous referees for their helpful comments. This research was funded by a fellowship of the Research Council of the Katholieke Universiteit Leuven, Belgium, the National Natural Science Foundation of China (Grant No. 10671078) and the Program for New Century Excellent Talents in University, the Ministry of Education of China.

\section{References}

1. Baker C T H, Ford N J. Some applications of the boundary-locus method and the method of D-partitions. IMA J Numer Anal, 1991, 11: 143-158

2. Baker C T H, Ford N J. Stability properties of a scheme for the approximate solution of a delay-integro-differential equations. Appl Numer Math, 1992, 9: 357370

3. Bellen A, Zennaro M. Numerical Methods for Delay Differential Equations. Oxford: Oxford University Press, 2003

4. Brunner H. Collocation Methods for Volterra Integral and Related Functional Equations. Cambridge Monographs on Applied and Computational Mathematics, Vol 15. Cambridge: Cambridge University Press, 2004

5. Brunner H, Hairer E, Norsett S P. Runge-Kutta theory for Volterra integral equations of the second kind. Math Comp, 1982, 39: 147-163

6. Brunner H, Lambert J D. Stability of numerical methods for Volterra integrodifferential equations. Computing, 1974, 12: 75-89

7. Calvo M, Grande T. On the asymptotic stability of $\Theta$-methods for delay differential equations. Numer Math, 1988, 54: 257-269

8. Cryer C W. Highly stable multistep methods for retarded differential equations. SIAM J Numer Anal, 1974, 11: 788-797

9. Diekmann O, Van Gils S A, Verduin Lunel S M, Walther H -O. Delay equations: Functional-, Complex-, and Nonlinear Analysis. Berlin: Springer-Verlag, 1995

10. Guglielmi N. On the asymptotic stability properties of Runge-Kutta methods for delay differential equations. Numer Math, 1997, 77: 467-485

11. Guglielmi N. Delay dependent stability regions of $\Theta$-methods for delay differential equations. IMA J Numer Anal, 1998, 18: 399-418

12. Guglielmi N. Asymptotic stability barriers for natural Runge-Kutta processes for delay equations. SIAM J Numer Anal, 2001, 39: 763-783

13. Guglielmi N, Hairer E. Order stars and stability for delay differential equations. Numer Math, 1999, 83: 371-383 
14. Guglielmi N, Hairer E. Geometric proofs of numerical stability for delay equations. IMA J Numer Anal, 2001, 21: 439-450

15. Hairer E, Wanner G. Solving Ordinary Differential equations II. Berlin: Springer, 1991

16. Hale J K, Verduyn Lunel S M. Introduction to Functional Differential Equations. New York: Springer-Verlag, 1993

17. Hu G, Mitsui T. Stability analysis of numerical methods for systems of neutral delay-differential equations. BIT, 1995, 35: 504-515

18. Huang C. Stability of linear multistep methods for delay integro-differential equations. Comput Math Appl, 2008, 55: 2830-2838

19. Huang C, Vandewalle S. An analysis of delay-dependent stability for ordinary and partial differential equations with fixed and distributed delays. SIAM J Sci Comput, 2004, 25: 1608-1632

20. in't Hout K J. Stability analysis of Runge-Kutta methods for systems of delay differential equations. IMA J Numer Anal, 1997, 17: 17-27

21. Koto T. A stability property of A-stable natural Runge-Kutta methods for systems of delay differential equations. BIT, 1994, 34: 262-267

22. Koto T. Stability of Runge-Kutta methods for delay integro-differential equations. J Comput Appl Math, 2002, 145: 483-492

23. Li S. Stability analysis of solutions to nonlinear stiff Volterra functional differential equations in Banach spaces. Sci China, Ser A, 2005, 48: 372-387

24. Lubich Ch. Runge-Kutta theory for Volterra integrodifferential equations. Numer Math, 1982, 40: 119-135

25. Luzyanina T, Engelborghs K, Roose D. Computing stability of differential equations with bounded distributed delays. Numer Algorithms, 2003, 34: 41-66

26. Maset S. Stability of Runge-Kutta methods for linear delay differential equations. Numer Math, 2000, 87: 355-371

27. Maset S. Instability of Runge-Kutta methods when applied to linear systems delay differential equations. Numer Math, 2002, 90: 555-562

28. Matthys J. A-stable linear multistep methods for Volterra integro-differential equations. Numer Math, 1976, 27: 85-94

29. Sidibe B S, Liu M Z. Asymptotic stability for Gauss methods for neutral delay differential equation. J Comput Math, 2002, 20: 217-224

30. $\mathrm{Wu} \mathrm{S}$, Gan S. Analytical and numerical stability of neutral delay integro-differential equations and neutral delay partial differential equations. Comput Math Appl, 2008, 55: 2426-2443

31. Zhang C, Vandewalle S. Stability criteria for exact and discrete solutions of neutral multidelay-integro-differential equations. Adv Comput Math, 2008, 28: 383-399

32. Zhao J J, Xu Y, Liu M Z. Stability analysis of numerical methods for linear neutral Volterra delay-integro-differential system. Appl Math Comput, 2005, 167: 10621079 